TRANSACTIONS OF THE

AMERICAN MATHEMATICAL SOCIETY

Volume 354, Number 8, Pages 2997-3009

S 0002-9947(02)02989-6

Article electronically published on March 29, 2002

\title{
THE BERGMAN METRIC ON A STEIN MANIFOLD WITH A BOUNDED PLURISUBHARMONIC FUNCTION
}

\author{
BO-YONG CHEN AND JIN-HAO ZHANG
}

\begin{abstract}
In this article, we use the pluricomplex Green function to give a sufficient condition for the existence and the completeness of the Bergman metric. As a consequence, we proved that a simply connected complete Kähler manifold possesses a complete Bergman metric provided that the Riemann sectional curvature $\leq-A / \rho^{2}$, which implies a conjecture of Greene and Wu. Moreover, we obtain a sharp estimate for the Bergman distance on such manifolds.
\end{abstract}

\section{INTRODUCTION}

Let $M$ be a complex $n$-dimensional manifold. Let $\mathcal{H}$ be the space of holomorphic $n$-forms on $M$ such that $\left|\int_{M} f \wedge \bar{f}\right|<\infty$. This space is a separable complex Hilbert space with an inner product $\left(f_{1}, f_{2}\right)=i^{n^{2}} \int_{M} f_{1} \wedge \bar{f}_{2}$. Let $h_{0}, h_{1}, \cdots$ be a complete orthonormal basis for $\mathcal{H}$. Then the $2 n$-form defined on $M \times M$ given by $K=\sum_{j=0}^{\infty} h_{j} \wedge \bar{h}_{j}$ is called the Bergman kernel form of $M$. Let $z=\left(z_{1}, \cdots, z_{n}\right)$ be a local coordinate system in $M$ and let $K(z)=K^{*}(z) d z_{1} \wedge \cdots \wedge d z_{n} \wedge d \bar{z}_{1} \wedge \cdots \wedge d \bar{z}_{n}$, where $K^{*}$ is a locally defined function. Then $\beta:=\partial \bar{\partial} \log K^{*}$ is a well-defined Hermitian form of bidegree $(1,1)$, whenever $K^{*}$ is nonzero. We say that $M$ possesses a Bergman metric iff $\beta$ is everywhere positive definite. In 1959, Kobayashi [11] began to investigate the completeness of the Bergman metric. After that, there are a lot of papers concerning the Bergman completeness for bounded pseudoconvex domains in $\mathbf{C}^{n}$ (see [3], [18] for a review). There are two general results: One says that any bounded hyperconvex domain is Bergman complete (cf. [1, [7]); the other states that any bounded pseudoconvex domain whose boundary can be locally described as the graph of a continuous function is also Bergman complete (cf. 4]). However, little is known for the Bergman metric of manifolds except the early work of Greene and $\mathrm{Wu}[6]$. They proved that a simply connected complete Kähler manifold possesses a complete Bergman metric if the sectional curvature is pinched between two negative constants, or the curvature is nonpositive and the

Received by the editors August 1, 2001.

2000 Mathematics Subject Classification. Primary 32H10.

Key words and phrases. Bergman metric, pluricomplex Green function, sectional curvature, Kähler manifold.

The first author was supported by an NSF grant TY10126005 and a grant from Tongji Univ. No. 1390104014

The second author was supported by project G1998030600.

(C)2002 American Mathematical Society 
following estimate holds outside a compact subset of $M$ :

$$
-\frac{B}{\rho^{2}} \leq \text { curvature } \leq-\frac{A}{\rho^{2}}
$$

for some positive constants $A, B$ (in this paper, curvature will mean sectional curvature). Here $\rho$ denotes the distance function relative to some fixed point $o$ in $M$. They conjectured that the lower estimate $-\frac{B}{\rho^{2}}$ is unnecessary. We will solve this conjecture in the present paper.

To formulate our results precisely, we need some notions. Let $M$ be any complex manifold. We denote by $P S H(M)$ the set of all plurisubharmonic (psh) functions on $M$. According to Klimek [10, we define the pluricomplex Green function with a logarithmic pole $y$ on $M$ by

$$
g_{M}(x, y)=\sup \{u(x)\},
$$

where the supremum is taken over all negative functions $u \in P S H(M)$ satisfying the property that the function $u-\log |z|$ is bounded from above in a deleted neighborhood of $y$ for some holomorphic local coordinates $z$ centered at $y$, that is, $z(y)=0$. It is known from [10] that for any $y \in M$ the function $g_{M}(\cdot, y)$ belongs to the above class, and it coincides with the classical (negative) Green function on hyperbolic Riemann surfaces (a Riemann surface is called hyperbolic if there is a negative nonconstant subharmonic function).

Definition. A complex manifold $M$ is said to satisfy the property $(B 1)$ if for any $y \in M$ there is a positive number $a>0$ such that the sublevel set $A(y, a):=$ $\left\{x \in M: g_{M}(x, y)<-a\right\}$ is relatively compact in $M$.

It is easy to see that any bounded domain $D$ in $\mathbf{C}^{n}$ has the property $(B 1)$ because of the trivial estimate $g_{D}(x, y) \geq \log \frac{|x-y|}{R_{D}}$, where $R_{D}$ is the diameter of $D$. We will also show in section 2 that any hyperbolic Riemann surface, any complex manifold carrying a bounded continuous strictly psh function, and any hyperconvex Stein manifold have the property $(B 1)$. Following Stehlé [17, we called a complex manifold $M$ hyperconvex if there exists a negative psh function $u$ such that the sublevel set $\{u<-c\}$ is relatively compact in $D$ for every $c>0$.

Definition. A complex manifold $M$ is said to satisfy the property (B2) if for any sequence of points $\left\{y_{k}\right\}, k=1,2, \cdots$, which has no adherent point in $M$ there exist a subsequence $\left\{y_{k_{j}}\right\}, j=1,2, \cdots$, and a number $a>0$ such that for any compact subset $K$ one has $A\left(y_{k_{j}}, a\right) \subset M \backslash K$ for all sufficiently large $j$.

Theorem 1. If $M$ is a Stein mainfold which satisfies the property (B1), then it possesses a Bergman metric. If furthermore, $M$ satisfies the property (B2), then the Bergman metric is complete.

With an application of Theorem 1, we solve the conjecture of Greene and Wu in the sequel.

Theorem 2. Let $M$ be a simply-connected complete Kähler manifold of dimension $n$ with nonpositive sectional curvature such that the inequality

$$
\text { curvature } \leq-\frac{A}{\rho^{2}}
$$

holds outside a compact subset of $M$ for a suitable positive constant $A$. Then $M$ possesses a complete Bergman metric. 
We also have the following consequences of Theorem 1:

Corollary 3. Any hyperconvex Riemann surface is Bergman complete.

Corollary 4. Let $D$ be a domain in $\mathbf{C}^{n}$, not necessarily bounded. Suppose that there exists a negative $C^{2}$ psh exhaustion function $\psi$ on $D$, such that

$$
\partial \bar{\partial} \psi \geq \partial \bar{\partial}|z|^{2} .
$$

Then $D$ is Bergman complete.

This domain was introduced by Sibony in [15], where he obtained an estimate of the Kobayashi metric for this domain.

In fact, this paper is a continuation of the paper 2], where the first-named author proved the Bergman completeness under the assumption of curvature $\leq-c$ for some positive constant. Greene and Wu used the geometry method of Siu and Yau [16] to get a comparison of the Bergman metric and the Kähler metric of the manifold which implies the completeness of the Bergman metric; hence the hypothesis that the curvature is bounded from below is essential. In this paper we just verify Kobayashi's criterion for Theorem 1 with help of the $L^{2}$ estimates of Hörmander type. Under the curvature condition of Theorem 2, Greene and $\mathrm{Wu}[6]$ constructed some special bounded psh exhaustion functions on the manifold. These functions enable us to show that the manifold satisfies the properties $(B 1)$ and $(B 2)$.

Using a recent result of Jost and Zuo [9] together with Theorem 1, we obtain a vanishing theorem for the $L^{2}$-cohomology groups with respect to the Bergman metric. Let $\left(M, d s^{2}\right)$ be a complete Kähler manifold of dimension $n$ and let $\mathcal{H}^{p, q}(M)$ denote the space of square-integrable harmonic $(p, q)$ forms on $M$. The result of Jost and Zuo says that if the sectional curvature is nonpositive, then $\mathcal{H}^{p, q}(M)=\{0\}$ for $p+q \neq n$. This implies the following

Corollary 5. Let $M$ be as in Theorem 1. Suppose that the sectional curvature of the Bergman metric is nonpositive. Then one has $\mathcal{H}_{\beta}^{p, q}(M)=\{0\}$ for $p+q \neq n$, where $\mathcal{H}_{\beta}^{p, q}(M)$ denotes the space of harmonic $(p, q)$ forms on $M$ which are squareintegrable with respect to the Bergman metric $\beta$.

In 1995, Diederich and Ohsawa [5] introduced a method of estimating the Bergman distance, which is based on Kobayashi's alternative definition of the Bergman metric. Inspired by their work, we are able to improve Theorem 2 as follows:

Theorem 6. Let $M$ be a simply-connected complete Kähler manifold of dimension $n$ with nonpositive sectional curvature.

1) If the inequality (1) holds outside a compact subset of $M$ for suitable positive constant $A$, then there exists a positive constant $C^{\prime}$ such that

$$
\operatorname{dist}_{\beta}(o, x) \geq C^{\prime} \log \rho(x),
$$

where dist ${ }_{\beta}(o, x)$ denotes the distance between o and $x$ with respect to the Bergman metric.

2) If the curvature is bounded from above by a negative constant $-A$, then

$$
\operatorname{dist}_{\beta}(o, x) \geq C^{\prime \prime} \rho(x)
$$

for a suitable constant $C^{\prime \prime}>0$. 


\section{Proof of Theorem 1}

We assume first that $M$ satisfies the property $(B 1)$, that is, for any $y \in M$ there is a number $a>0$ so that $A(y, a) \subset \subset M$. To prove the existence of the Bergman metric, it suffices to show, according to [11], the following two statements:

(i) Given any point $y$ of $M$, there exists a form $f \in \mathcal{H}$ such that $f(y) \neq 0$.

(ii) For any holomorphic vector $X$ at $y$, there exists a form $f \in \mathcal{H}$ such that $f^{*}(0)=0$ and $X f^{*}(0) \neq 0$, where $f(z)=f^{*}(z) d z_{1} \wedge \cdots \wedge d z_{n}$ in a local coordinate system centered at $y$.

Since $M$ is Stein, there exist $n$ holomorphic functions $\zeta_{1}, \cdots, \zeta_{n}$ on $M$ which form a local coordinate system centered at $y$. Without loss of generality, we assume $X=\partial / \partial \zeta_{1}$. We take a cut-off function $\chi: \mathbf{R} \rightarrow[0,1]$ such that $\chi \equiv 1$ on $(-\infty,-1]$ and $\chi \equiv 0$ on $[0, \infty)$. We set

$$
\eta= \begin{cases}\chi\left(-\log \left(-g_{M}(\cdot, y)+a\right)+\log (2 a)\right) & \text { for case }(i) \\ \chi\left(-\log \left(-g_{M}(\cdot, y)+a\right)+\log (2 a)\right) \zeta_{1} & \text { for case }(i i)\end{cases}
$$

and

$$
\varphi=2(n+1) g_{M}(\cdot, y)-\log \left(-g_{M}(\cdot, y)+a\right) .
$$

Clearly $\varphi \in P S H(M)$. We will show that there exists a constant $C=C(y, a)$ so that the equation $\bar{\partial} u=\bar{\partial} \eta \wedge d \zeta_{1} \wedge \cdots \wedge d \zeta_{n}$ has a solution in the distribution sense such that the following inequality holds:

$$
\left|\int_{M} u \wedge \bar{u} e^{-\varphi}\right| \leq C
$$

If we have proved the above fact under the assumption that the function $g_{M}(\cdot, y)$ is $C^{\infty}$, then for the general case, we can exhaust $M$ by an increasing sequence of relatively compact Stein domains $M_{j}, j=1,2, \cdots$, and for each $j$ the psh function $g_{M}(\cdot, y)$ can be approximated uniformly on $\bar{M}_{j}$ by negative strictly psh functions $\psi_{j, k}, k=1,2, \cdots$. We replace $g_{M}(\cdot, y)$ by $\psi_{j, k}$. It follows that there is a solution to the equation $\bar{\partial} u_{j, k}=\bar{\partial} \eta \wedge d \zeta_{1} \wedge \cdots \wedge d \zeta_{n}$ on $M_{j}$ together with the estimate

$$
\left|\int_{M_{j}} u_{j, k} \wedge \bar{u}_{j, k} e^{-\varphi}\right| \leq C
$$

for a suitable constant $C>0$ depending only on $y$ and $a$. To obtain the desired solution, we only need to take a weak limit as $k, j \rightarrow \infty$. This limiting procedure is standard (cf. [4]). Hence we may assume $g_{M}(\cdot, y)$ is $C^{\infty}$. Next, we need an $L^{2}$ estimate of Hörmander type for the $\bar{\partial}$-equation on complete Kähler manifolds.

Proposition 7 (cf. 13]). Let $M$ be a complete Kähler manifold and let $\varphi$ be a $C^{\infty}$ strictly psh function on $M$. Then for any $\bar{\partial}$-closed $(n, 1)$ form with $\int_{M}|v|_{\partial \bar{\partial} \varphi}^{2} e^{-\varphi} d V_{\varphi}$ $<\infty$, there is an $n$-form $u$ on $M$ such that $\bar{\partial} u=v$ and

$$
\left|\int_{M} u \wedge \bar{u} e^{-\varphi}\right| \leq \int_{M}|v|_{\partial \bar{\partial} \varphi}^{2} e^{-\varphi} d V_{\varphi}
$$

where $d V_{\varphi}$ denotes the volume with respect to $\partial \bar{\partial} \varphi$. 
This proposition gives us a solution to the equation $\bar{\partial} u=\bar{\partial} \eta \wedge d \zeta_{1} \wedge \cdots \wedge d \zeta_{n}$ with the following inequality:

$$
\begin{aligned}
\left|\int_{M} u \wedge \bar{u} e^{-\varphi}\right| & \leq \int_{M}|\bar{\partial} \eta|_{\partial \bar{\partial} \varphi}^{2} e^{-\varphi} d V_{\varphi} \\
& \leq C_{1},
\end{aligned}
$$

noting that $|\bar{\partial} \chi(\cdot)|_{\partial \bar{\partial} \varphi} \leq \sup \left|\chi^{\prime}\right|$ because

$$
\partial \bar{\partial} \varphi \geq-\partial \bar{\partial} \log \left(-g_{M}(\cdot, y)+a\right) \geq \partial \log \left(-g_{M}(\cdot, y)+a\right) \bar{\partial} \log \left(-g_{M}(\cdot, y)+a\right) .
$$

Here $C_{1}$ is a positive constant depending only on $y, a$ and the choice of $\chi ;|\cdot|_{\partial \bar{\partial} \varphi}$ denotes the point norm w.r.t. the metric $\partial \bar{\partial} \varphi$. This implies that the form $f:=$ $\eta \wedge d \zeta_{1} \wedge \cdots \wedge d \zeta_{n}-u \in \mathcal{H}$, because $\varphi$ is bounded from above. Moreover, the singularity of $\varphi$ shows that $f^{*}(0)=1$ for case (i), while $f^{*}(0)=0, \partial f^{*} / \partial \zeta_{1}(0)=1$ for case (ii). This completes the first part of the proof.

To prove the second part, we need the criterion of Kobayashi for Bergman completeness:

Proposition 8 (cf. [12]). Let $M$ be a complex manifold which possesses a Bergman metric. Assume that there exists a dense subspace $\mathcal{H}^{\prime}$ of $\mathcal{H}$ such that for any $f \in \mathcal{H}^{\prime}$ and for any sequence of points $\left\{y_{k}\right\}_{k=1}^{\infty}$ of $M$ which has no adherent point in $M$, there is a subsequence $\left\{y_{k_{j}}\right\}_{j=1}^{\infty}$ such that

$$
\lim _{j \rightarrow \infty} \frac{f\left(y_{k_{j}}\right) \wedge \bar{f}\left(y_{k_{j}}\right)}{K\left(y_{k_{j}}\right)}=0 .
$$

Then $M$ is Bergman complete.

Let $f \in \mathcal{H}$, and let $\left\{y_{k}\right\}_{k=1}^{\infty}$ be a sequence of points which has no adherent point in $M$. For any $\epsilon>0$ one can find a compact subset $M_{\epsilon}$ of $M$ such that

$$
\left|\int_{M \backslash M_{\epsilon}} f \wedge \bar{f}\right|<\epsilon .
$$

By the hypothesis $(B 2)$, one can find a subsequence $\left\{y_{k_{j}}\right\}$ with the following property: there exists a positive number $a$ (independent of $\epsilon$ and $j$ ) such that $A\left(y_{k_{j}}, a\right) \subset M \backslash M_{\epsilon}$ for all sufficiently large $j$. Let $\chi$ be as before, and set

$$
\begin{aligned}
\eta_{j} & =\chi\left(-\log \left(-g_{M}\left(\cdot, y_{k_{j}}\right)+a\right)+\log (2 a)\right) f, \\
\varphi_{j} & =2 n g_{M}\left(\cdot, y_{k_{j}}\right)-\log \left(-g_{M}\left(\cdot, y_{k_{j}}\right)+a\right) .
\end{aligned}
$$

We can solve the equation $\bar{\partial} u_{j}=\bar{\partial} \eta_{j}$ essentially as above together with the following estimate:

$$
\begin{aligned}
\left|\int_{M} u_{j} \wedge \bar{u}_{j} e^{-\varphi_{j}}\right| & \leq \int_{M}\left|\bar{\partial} \eta_{j}\right|_{\partial \bar{\partial} \varphi_{j}}^{2} e^{-\varphi_{j}} d V_{\varphi_{j}} \\
& \leq C_{2}\left|\int_{\operatorname{supp} \bar{\partial} \eta_{j}} f \wedge \bar{f}\right| \\
& \leq C_{2} \epsilon,
\end{aligned}
$$

because supp $\bar{\partial} \eta_{j} \subset A\left(y_{k_{j}}, a\right) \subset M \backslash M_{\epsilon}$ for $j$ sufficiently large. Here $C_{2}$ is a constant depending only on $a$ and the choice of $\chi$. We set $f_{j}=\eta_{j}-u_{j}$. Then $f_{j}\left(y_{k_{j}}\right)=f\left(y_{k_{j}}\right)$ 
and $\left|\int_{M} f_{j} \wedge \bar{f}_{j}\right| \leq C_{3} \epsilon$. It follows that

$$
\frac{f\left(y_{k_{j}}\right) \wedge \bar{f}\left(y_{k_{j}}\right)}{K\left(y_{k_{j}}\right)} \leq\left|\int_{M} f_{j} \wedge \bar{f}_{j}\right| \leq C_{3} \epsilon .
$$

Hence the assertion follows immediately from Proposition 8 .

Let us see that there are various complex manifolds satisfying $(B 1)$ :

1. $D$ is a hyperbolic Riemann surface, that is, $M$ carries a bounded non-constant subharmonic function. It is well known that this condition is equivalent to the fact that $M$ carries a (negative) Green function. Since $g_{M}(x, y)$ is harmonic in $M \backslash\{y\}$ and $g_{M}(x, y)-\log |z|$ is harmonic in a local coordinate chart at $y$, we see that $M$ satisfies the property $(B 1)$.

2. Let $M$ be a complex manifold carrying a bounded continuous strictly psh function $\psi$. By the well-known theorem of Richberg [14, we may assume that $\psi$ is $C^{\infty}$. For any $y \in M$, we take a function $\kappa$ which is compactly supported in a coordinate chart at $y$ and identically equal to 1 in a neighborhood of $y$. One can find a constant $a_{y}$ such that $\kappa \log |z|+a_{y} \psi$ is a psh function on $M$ wth a logarithmic pole at $z(y)=0$ which is bounded above by a constant depending only on $y$. It follows from the definition of the pluricomplex Green function that $M$ satisfies $(B 1)$.

3. Let $M$ be a hyperconvex Stein manifold. We will show that $M$ satisfies $(B 1)$. We first prove the following fact.

Claim. Let $M$ be a Stein manifold and let $y \neq y^{\prime}$ be two points of $M$. Then there is a holomorphic function $f$ on $M$ such that $f(y)=0, d f(y)=0$ and $f\left(y^{\prime}\right)=1$.

Proof. The proof is standard. Let $\psi$ be a $C^{\infty}$ strictly psh exhaustion function on $M$. Similarly as before, one can find psh functions $\psi_{y}, \psi_{y^{\prime}}$ on $M$ with a logarithmic pole at $y, y^{\prime}$ respectively. We choose a cutoff function $\tau$ which is compactly supported in $M$ and is such that $\tau \equiv 0$ in a neighborhood of $y$ and $\tau \equiv 1$ in a neighborhood of $y^{\prime}$. Now we take a convex, rapidly increasing function $\gamma$ such that there is, according to Theorem 5.2.4 in [8], a solution to the equation $\bar{\partial} u=\bar{\partial} \tau$ satisfying the following estimate:

$$
\int_{M}|u|^{2} e^{-\varphi} d V \leq \int_{M}|\bar{\partial} \tau|^{2} e^{-\varphi} d V \leq \widetilde{C}
$$

where $\varphi=\gamma \circ \psi+2(n+1) \psi_{y}+2 n \psi_{y^{\prime}}$; the point norm and the volume are associated to some fixed Kähler metric on $M$. Then the function defined by $f=\tau-u$ is holomorphic on $M$ and satisfies $f(y)=0, d f(y)=0$ and $f\left(y^{\prime}\right)=1$.

Now let $\mu$ be a negative psh exhaustion function on $M$. Again we take $n$ globally defined holomorphic functions $\zeta_{1}, \cdots, \zeta_{n}$ which form a local coordinate system centered at $y$, and denote by $U$ the coordinate neighborhood of $y$. We set

$$
K=\{x \in M: \mu(x) \leq \mu(y) / 8\} \backslash U .
$$

Since it is compact in $M$, we obtain from the above claim finite holomorphic functions $\zeta_{n+1}, \cdots, \zeta_{n+m}$ on $M$ such that $\zeta_{n+j}(y)=0, d \zeta_{n+j}(y)=0$ for all $1 \leq$ $j \leq m$, and the function $\sum_{j=1}^{m}\left|\zeta_{n+j}\right|^{2}$ is nowhere vanishing on $K$. We denote $\zeta=\left(\zeta_{1}, \cdots, \zeta_{n+m}\right)$ and set

$$
\begin{aligned}
\lambda & =\inf _{\{\mu(x)=\mu(y) / 2\}} \log |\zeta(x)| / R_{y} \\
\widetilde{\mu}(x) & =\lambda \frac{\log (-\mu(x)-\mu(y) / 4)-\log (-\mu(y) / 2)}{\log 3 / 2},
\end{aligned}
$$


where

$$
R_{y}=\sup _{\{\mu(x)=\mu(y) / 2\}}|\zeta(x)|+1 .
$$

It follows that $\widetilde{\mu}$ is a psh function on $M$ satisfying

$$
\begin{array}{ll}
\widetilde{\mu}(x)=\lambda \leq \log |\zeta(x)| / R_{y} & \text { if } \mu(x)=\mu(y) / 2, \\
\widetilde{\mu}(x)=0 \geq \log |\zeta(z)| / R_{y} & \text { if } \mu(x)=\mu(y) / 4 .
\end{array}
$$

Hence the function defined by

$$
v(x)= \begin{cases}\log |\zeta(x)| / R_{y} & \text { if } \mu(x)<\mu(y) / 2 \\ \max \left\{\log |\zeta(x)| / R_{z}, \widetilde{\mu}(x)\right\} & \text { if } \mu(y) / 2 \leq \mu(x) \leq \mu(y) / 4, \\ \widetilde{\mu}(x) & \text { if } \mu(x)>\mu(y) / 4\end{cases}
$$

is a psh function on $M$ with a logarithmic pole at $y$ which is bounded from above by a constant depending only on $y$. Then, similarly as above, $M$ satisfies $(B 1)$.

Proof of Corollary 3. It suffices to show that $M$ satisfies (B2). Let $\mu$ be a negative psh exhaustion function on $M$. We denote

$$
M_{c}=\{x \in M: \mu(x)<-c\}
$$

for any $c>0$. Now let $c$ be fixed and let $y \in M_{2 c}$ be arbitrary. We set

$$
\psi_{y}(x)= \begin{cases}\max \left\{C \mu(x), g_{M}(x, y)-1\right\} & \text { if } x \in M \backslash M_{c}, \\ g_{M}(x, y)-1 & \text { if } x \in M_{c}\end{cases}
$$

where

$$
C=-c^{-1} \min _{\{\mu(x)=-c\}}\left(g_{M}(x, y)-1\right)
$$

is a constant depending only on $c$ because $g_{M}$ is a continuous function off the diagonal on $M \times M$. Clearly, $\psi_{y}$ is a negative psh function with a logarithnic pole at $y$, and furthermore, there is a positive constant $c^{\prime}<<c$ such that $\psi_{y}(x) \geq-1$ on $M \backslash M_{c^{\prime}}$. It follows from the extremal property of the Green function that the inequality $g_{M}(x, y) \geq-1$ holds there. Since $g_{M}$ is symmetric, we have

$$
g_{M}(x, y) \geq-1, \forall x \in M_{2 c}, y \in M \backslash M_{c^{\prime}} .
$$

It follows that $A(y,-1) \subset M \backslash M_{2 c}$ for any $y \in M \backslash M_{c^{\prime}}$, which implies the property (B2). The proof is complete.

Proof of Corollary 4. Since $\psi$ is a negative $C^{2}$ strictly psh function on $D$, according to the above facts, $D$ carries a Bergman metric. Moreover, it is the standard Bergman metric since $D$ is a domain in $\mathbf{C}^{n}$. Let $\left\{y_{k}\right\}$ be an arbitrary sequence of points which has no adherent point in $D$. We distinguish two cases:

(a) There is a subsequence $\left\{y_{k_{j}}\right\}$ such that $\left|y_{k_{j}}\right| \rightarrow \infty$ as $j \rightarrow+\infty$. We take a cutoff finction $\chi: \mathbf{R} \rightarrow[0,1]$ such that $\left.\chi\right|_{(-\infty, 1 / 2]}=1$ and $\left.\chi\right|_{[1,+\infty)}=0$. Since $\partial \bar{\partial} \psi \geq \partial \bar{\partial}|z|^{2}$, there is a constant $C^{\prime}>0$, depending only on the choice of $\chi$, such that the function $\varphi_{j}:=C^{\prime} \psi+\chi\left(\left|z-y_{k_{j}}\right|\right) \log \left|z-y_{k_{j}}\right|$ is a negative psh function on $D$ with a logarithmic pole at $y_{k_{j}}$. Hence for any compact subset $K$ of $D$, one has $A\left(y_{k_{j}},-1\right) \subset D \backslash K$ for all sufficiently large $j$. Similarly as in the proof of Theorem 1 , the criterion of Kobayashi holds for $\left\{y_{k}\right\}$.

(b) Otherwise, there is a subsequence $\left\{y_{k_{j}}\right\}$ such that $y_{k_{j}}$ converges to a boundary point $y_{0}$. Take a ball $B\left(y_{0}, 1\right)$ and set $D^{\prime}=D \cap B\left(y_{0}, 1\right)$. Clearly $D^{\prime}$ is a bounded 
hyperconvex domain. Without loss of generality, we may assume $y_{k_{j}} \in B\left(y_{0}, 1 / 4\right)$. If we have proved that

$$
K_{D}\left(y_{k_{j}}\right) \geq C^{\prime \prime} K_{D^{\prime}}\left(y_{k_{j}}\right)
$$

for some constant $C^{\prime \prime}$ independent of $j$, then for any $f \in \mathcal{H}(D)$

$$
\lim _{k \rightarrow \infty} \frac{\left|f\left(y_{k_{j}}\right)\right|^{2}}{K_{D}\left(y_{k_{j}}\right)} \leq \frac{1}{C^{\prime \prime}} \lim _{k \rightarrow \infty} \frac{\left|f\left(y_{k_{j}}\right)\right|^{2}}{K_{D^{\prime}}\left(y_{k_{j}}\right)}=0
$$

where the last equality was shown in [1], [7]. Hence Kobayashi's criterion holds for $\left\{y_{k}\right\}$. The proof is reduced to showing the localization property of the Bergman kernel. Let $\varphi_{j}$ be as above. We solve the equation

$$
\bar{\partial} u_{j}=\bar{\partial} \chi\left(\left|z-y_{0}\right|\right) K_{D^{\prime}}\left(z, y_{k_{j}}\right) / K_{D^{\prime}}^{1 / 2}\left(y_{k_{j}}\right)
$$

together with the following estimate:

$$
\int_{D}\left|u_{j}\right|^{2} e^{-2 n \varphi_{j}-\psi} d V \leq \int_{D}\left|\bar{\partial} \chi\left(\left|z-y_{0}\right|\right)\right|_{\partial \bar{\partial} \psi}^{2} e^{-2 n \varphi_{j}-\psi} d V \leq C^{\prime \prime \prime},
$$

where $C^{\prime \prime \prime}$ is a constant independent of $j$. Set again

$$
f_{j}=\chi\left(\left|z-y_{0}\right|\right) K_{D^{\prime}}\left(z, y_{k_{j}}\right) / K_{D^{\prime}}^{1 / 2}\left(y_{k_{j}}\right)-u_{j} .
$$

We obtain

$$
K_{D}\left(y_{k_{j}}\right) \geq \frac{\left|f\left(y_{k_{j}}\right)\right|^{2}}{\int_{D}|f|^{2} d V} \geq C^{\prime \prime} K_{D^{\prime}}\left(y_{k_{j}}\right)
$$

for a suitable constant $C^{\prime \prime}$ independent of $j$.

The proof follows immediately from Proposition 8.

\section{Proof of Theorem 2}

We will follow the argument of Greene and $\mathrm{Wu} 6$ throughout this section. Let $M$ be as in Theorem 1. Suppose that (1) holds in $M \backslash B(o, c)$ for some positive constant $c$, where $B(x, \delta)$ denotes the geodesic ball with radius $\delta$ around $x$. Let $x_{0}$ be any point in $M \backslash B(o, 2 c)$. Let $\rho_{0}$ denote the distance function relative to $x_{0}$. Let $G$ be the complete Kähler metric of $M$ and let $\mathrm{K}_{G}(x)$ denotes the maximum of the sectional curvatures at $x$. It is not difficult to see that the inequality

$$
\mathrm{K}_{G}(x) \leq-\frac{A}{4 \rho_{0}(x)^{2}}
$$

holds for all $x \in M \backslash B\left(x_{0}, 2 \rho\left(x_{0}\right)\right)$. Consider the new Kähler metric $H=\frac{G}{\rho\left(x_{0}\right)^{2}}$. Let $\gamma_{0}$ denote the distance function of $H$ relative to $x_{0}$. Then $\gamma_{0}=\frac{\rho_{0}}{\rho\left(x_{0}\right)}$ and $\mathrm{K}_{H}=\rho\left(x_{0}\right)^{2} \mathrm{~K}_{G}$. Hence inequality (3) is equivalent to

$$
\mathrm{K}_{H}(x) \leq-\frac{A}{4 \gamma_{0}(x)^{2}}
$$

for all $x \in M$ with $\gamma_{0}(x) \geq 2$. Notice also that $\mathrm{K}_{H} \leq 0$ everywhere. By Lemma 5.15 in [6], there is a complete Hermitian metric $h$ on the unit disc $D$ which is rotationally symmetric, and its Gaussian curvature $\mathrm{K}_{h}$ satisfies (a) $\mathrm{K}_{h} \leq 0$ and (b) if $\widetilde{\rho}$ denotes the distance function of $h$ relative to the origin, then

$$
\mathrm{K}_{h}= \begin{cases}0 & \text { on }\{\widetilde{\rho} \leq 2\} \\ -A /\left(4 \widetilde{\rho}^{2}\right) & \text { on }\{\widetilde{\rho} \geq 3\}\end{cases}
$$


and in the annulus $\{2<\widetilde{\rho}<3\}, \mathrm{K}_{h}$ is rotationally symmetric. Write $h=d \widetilde{\rho}^{2}+$ $f(\widetilde{\rho})^{2} d \theta^{2}$ in terms of geodesic polar coordinates. Since $f^{\prime \prime}=-\mathrm{K}_{h} f$, it follows that $f^{\prime \prime} \equiv 0$ on $[0,2]$; hence $f(\widetilde{\rho})=\widetilde{\rho}$ there. Next we write $h$ as follows:

$$
h=\eta(r) d z d \bar{z}=\eta(r)\left(d r^{2}+r^{2} d \theta^{2}\right),
$$

where $r: D \rightarrow[0,1)$ is the ordinary radial function on $D$. Clearly, one has

$$
\begin{aligned}
\eta(r) & =[\widetilde{\rho}(r)]^{2}, \\
r^{2} \eta(r) & =f(\widetilde{\rho}(r))^{2} .
\end{aligned}
$$

We will regard $r$ as a function $\widetilde{\rho}$ so that $r:[0, \infty) \rightarrow[0,1)$. By (3), (4), one has

$$
\frac{1}{r}=\frac{\widetilde{\rho}^{\prime}(r)}{f(\widetilde{\rho}(r))}
$$

Integrating both sides relative to $d r$ from $r$ to 1 , we obtain

$$
\begin{aligned}
r(\widetilde{\rho}) & =\exp \left\{-\int_{r}^{1} \frac{\widetilde{\rho}^{\prime}(r)}{f(\widetilde{\rho}(r))} d r\right\} \\
& =\exp \left\{-\int_{\widetilde{\rho}}^{\infty} \frac{1}{f}\right\} .
\end{aligned}
$$

Set $\phi_{x_{0}}=r\left(\gamma_{0}\right)^{2}$. Using a Hessian comparison theorem, Greene and Wu proved that $\phi_{x_{0}}$ is a bounded exhaustion function on $M$ which is $C^{\infty}$ strictly psh, and satisfies $0 \leq \phi_{x_{0}}<1, \phi_{x_{0}}^{-1}(0)=x_{0}, \phi_{x_{0}}=O\left(\gamma_{0}^{2}\right)$ near $x_{0}$, and $\log \phi_{x_{0}}$ is also psh. Observe that

$$
\begin{aligned}
\log \phi_{x_{0}}(x) & =2 \log r\left(\gamma_{0}(x)\right)=-2 \int_{\gamma_{0}(x)}^{\infty} \frac{1}{f} \\
& =-2\left(\int_{\gamma_{0}(x)}^{1} \frac{1}{f}+\int_{1}^{\infty} \frac{1}{f}\right)=2 \log \frac{\gamma_{0}(x)}{b}
\end{aligned}
$$

for any $x \in M$ with $\gamma_{0}(x) \leq 1$, since $f(t)=t$ for $t \leq 2$. Here $b=\exp \left(\int_{1}^{\infty} \frac{1}{f}\right)>1$, which is a constant depending only on $A$. On the other hand, one has

$$
\log \phi_{x_{0}}(x) \geq-2 \int_{1}^{\infty} \frac{1}{f}=-2 \log b
$$

whenever $\gamma_{0}(x)>1$. If we set

$$
\widetilde{A}\left(x_{0}, c\right):=\left\{x \in M: \log \phi_{x_{0}}(x)<-c\right\}
$$

for any $c>0$, then we immediately obtain the following fact.

Lemma 9. Under the condition of Theorem 2, one has

$$
\begin{aligned}
\widetilde{A}\left(x_{0}, 2 \log (2 b)\right) & \subset\left\{x \in M: \gamma_{0}(x)<\frac{1}{2}\right\} \\
& =\left\{x \in M: \rho_{0}(x)<\frac{1}{2} \rho\left(x_{0}\right)\right\}
\end{aligned}
$$

for any $x_{0} \in M \backslash B(o, 2 c)$. 
Proof of Theorem 2. For any $x_{0} \in M \backslash B(o, 2 c), \phi_{x_{0}}-1$ is a negative $C^{\infty}$ strictly psh exhaustion function of $M$. It follows from the previous section that $M$ satisfies the property $(B 1)$. By Lemma 9 we claim that, for any sequence of points $y_{k}, k=$ $1,2, \cdots$, which has no adherent point in $M$,

$$
\begin{aligned}
& A\left(y_{k}, 2 \log (2 b)\right) \subset \widetilde{A}\left(y_{k}, 2 \log (2 b)\right) \\
\subset \quad & \left\{x \in M: \rho_{k}(x)<\frac{1}{2} \rho\left(y_{k}\right)\right\} \subset\left\{x \in M: \rho(x)>\frac{1}{2} \rho\left(y_{k}\right)\right\}
\end{aligned}
$$

provided $k$ is sufficiently large. Here $\rho_{k}$ denotes the distance associated to $y_{k}$. This implies that the property (B2) is also satisfied. Thus the assertion follows immediately from Theorem 1.

\section{Proof of Theorem 6}

We first prove 1$)$. Let $x_{1}, x_{2}$ be two arbitrary points which satisfy $\rho\left(x_{2}\right) \geq 2 c$ and $\rho\left(x_{1}\right)=4 \rho\left(x_{2}\right)$. Take a complete orthonormal basis $\left\{h_{j}\right\}_{j=0}^{\infty}$ for $\mathcal{H}$ such that $h_{j}\left(x_{2}\right)=0$ for all $j \geq 1$. We claim that the following holds:

Lemma 10. There is a constant $C_{4}>0$ such that

$$
\begin{aligned}
& C_{4} h_{0}\left(x_{1}\right) \wedge \bar{h}_{0}\left(x_{1}\right) \\
\leq & \sup \left\{f\left(x_{1}\right) \wedge \bar{f}\left(x_{1}\right): f \in \mathcal{H}, f\left(x_{2}\right)=0,\left|\int_{M} f \wedge \bar{f}\right| \leq 1\right\},
\end{aligned}
$$

where for any two forms $f(z)=f^{*}(z) d z_{1} \wedge \cdots \wedge d z_{n}, g(z)=g^{*}(z) d z_{1} \wedge \cdots \wedge d z_{n}$, $f(z) \wedge \bar{f}(z) \leq g(z) \wedge \bar{g}(z)$ iff $\left|f^{*}(z)\right| \leq\left|g^{*}(z)\right|$.

Proof. We will use Lemma 9 with $x_{0}=x_{1}, x_{2}$ respectively. Set

$$
\varphi=n\left(\log \phi_{x_{1}}+\log \phi_{x_{2}}\right)+\phi_{x_{1}}-\log \left(-\log \frac{\phi_{x_{1}}}{2}\right) .
$$

Clearly, it is a $C^{\infty}$ strictly psh function on $M \backslash\left\{x_{1}, x_{2}\right\}$ which satisfies the following estimate:

$$
\partial \bar{\partial} \varphi \geq \partial \bar{\partial}\left(-\log \left(-\log \frac{\phi_{x_{1}}}{2}\right)\right) \geq \frac{\partial \log \phi_{x_{1}} \bar{\partial} \log \phi_{x_{1}}}{\left(\log \frac{\phi_{x_{1}}}{2}\right)^{2}} .
$$

Choose a $C^{\infty}$ cutoff function $\chi: \mathbf{R} \rightarrow[0,1]$ such that $\left.\chi\right|_{(-\infty,-2)}=1,\left.\chi\right|_{(-1, \infty)}=0$. Set

$$
\eta=\chi\left(\frac{\log \phi_{x_{1}}}{2 \log (2 b)}\right) h_{0}
$$

Clearly, $\eta\left(x_{1}\right)=h_{0}\left(x_{1}\right)$, and it follows from Lemma 9 that

$$
\begin{aligned}
\operatorname{supp} \eta & \subset\left\{x \in M: \log \phi_{x_{1}}(x)<-2 \log (2 b)\right\} \\
& \subset\left\{x \in M: \rho_{1}(x) \leq \frac{1}{2} \rho\left(x_{1}\right)\right\},
\end{aligned}
$$

where $\rho_{1}(x)$ denotes the distance function relative to $x_{1}$. It follows that $\eta\left(x_{2}\right)=0$ and

$$
\log \phi_{x_{2}}(x) \geq-2 \log (2 b), \quad \forall x \in \operatorname{supp} \eta,
$$

because $\rho\left(x_{1}\right)=4 \rho\left(x_{2}\right)$. By $(7)$, one has

$$
\left|\bar{\partial} \chi\left(\frac{\log \phi_{x_{1}}}{2 \log (2 b)}\right)\right|_{\partial \bar{\partial} \varphi} \leq C_{5},
$$


where $|\cdot|_{\partial \bar{\partial} \varphi}$ denotes the point norm with respect to the metric $\partial \bar{\partial} \varphi$ and $C_{6}>0$ is a constant that only depends on $b$ and the choice of $\chi$.

Observe that $M \backslash\left\{x_{1}, x_{2}\right\}$ still carries a complete Kähler metric, defined as follows:

$$
\partial \bar{\partial}\left(-\log \left(-\log \phi_{x_{1}}\right)-\log \left(-\log \phi_{x_{2}}\right)+\phi_{x_{1}}\right) .
$$

By Proposition 7, there is a solution to the equation $\bar{\partial} u=\bar{\partial} \eta$ on $M \backslash\left\{x_{1}, x_{2}\right\}$ which satisfies

$$
\begin{aligned}
\left|\int_{M \backslash\left\{x_{1}, x_{2}\right\}} u \wedge \bar{u} e^{-\varphi}\right| & \leq \int_{M \backslash\left\{x_{1}, x_{2}\right\}}|\bar{\partial} \eta|_{\partial \bar{\partial} \varphi}^{2} e^{-\varphi} d V_{\varphi} \\
& \leq\left.\left|\int_{M \backslash\left\{x_{1}, x_{2}\right\}}\right| \bar{\partial} \chi\left(\frac{\log \phi_{x_{1}}}{2 \log (2 b)}\right)\right|_{\partial \bar{\partial} \varphi} ^{2} h_{0} \wedge \overline{h_{0}} e^{-\varphi} \mid \\
& \leq C_{6}
\end{aligned}
$$

because of (8)-(10). Set $f=\eta-u$. It is a holomorphic $n$-form on $M \backslash\left\{x_{1}, x_{2}\right\}$, and by (11), $f$ can be extended holomorphically across $x_{1}, x_{2}$; moreover, $f\left(x_{1}\right)=$ $h_{0}\left(x_{1}\right), f\left(x_{2}\right)=0$ because of the singularity of $\varphi$ at $x_{1}, x_{2}$. Since $\varphi$ is bounded from above on $M$, one has

$$
\begin{aligned}
\left|\int_{M} f \wedge \bar{f}\right| & \leq 2\left|\int_{M} \eta \wedge \bar{\eta}\right|+2\left|\int_{M} u \wedge \bar{u}\right| \\
& \leq 2\left|\int_{M} h_{0} \wedge \bar{h}_{0}\right|+C_{7}\left|\int_{M} u \wedge \bar{u} e^{-\varphi}\right| \\
& \leq C_{8}
\end{aligned}
$$

from which the assertion immediately follows with the constant $C_{4}=C_{8}^{-1}$.

We proceed to prove the theorem. According to Kobayashi's alternative definition of the Bergman metric, $\beta$ is nothing but the pullback of the Fubini-Study metric of the infinite-dimensional complex projective space $\mathbf{C P}(\mathcal{H})$ (cf. [11). It follows that the Bergman distance $\operatorname{dist}_{\beta}\left(x_{1}, x_{2}\right)$ is no less than the Fubini-Study distance between the points $p_{1}=\left(a_{0}: a_{1}: \cdots\right)$ and $p_{2}=(1: 0: \cdots)$, where the $a_{j}$ are given by

$$
\left|a_{j}\right|^{2}=\frac{h_{j}\left(x_{1}\right) \wedge \bar{h}_{j}\left(x_{1}\right)}{\sum_{j=0}^{\infty} h_{j}\left(x_{1}\right) \wedge \bar{h}_{j}\left(x_{1}\right)}=\frac{h_{j}\left(x_{1}\right) \wedge \bar{h}_{j}\left(x_{1}\right)}{K\left(x_{1}\right)} .
$$

This implies that

$$
\operatorname{dist}_{\beta}\left(x_{1}, x_{2}\right) \geq \sqrt{\left|1-a_{0}\right|^{2}+\sum_{j=1}^{\infty}\left|a_{j}\right|^{2}} .
$$

Assume that the supremum on the right side of (5) is realized by a certain $n$-form $f$. Then, without loss of generality, we can take $h_{1}=f$. If $\left|a_{0}\right| \geq 1 / 2$, we have

$$
\begin{aligned}
\operatorname{dist}_{\beta}\left(x_{1}, x_{2}\right) & \geq\left|a_{1}\right|=\sqrt{\frac{f\left(x_{1}\right) \wedge \bar{f}\left(x_{1}\right)}{K\left(x_{1}\right)}} \\
& \geq \sqrt{C_{4}} \sqrt{\frac{h_{0}\left(x_{1}\right) \wedge \bar{h}_{0}\left(x_{1}\right)}{K\left(x_{1}\right)}}=\sqrt{C_{4}}\left|a_{0}\right| \geq \frac{1}{2} \sqrt{C_{4}} .
\end{aligned}
$$


Otherwise, it is clear that $\operatorname{dist}_{\beta}\left(x_{1}, x_{2}\right) \geq 1-\left|a_{0}\right| \geq \frac{1}{2}$. Therefore, there is a positive constant $C_{9}>0$ such that

$$
\operatorname{dist}_{\beta}\left(x_{1}, x_{2}\right) \geq C_{9}
$$

holds for any $x_{1}, x_{2} \in M$ satisfying $\rho\left(x_{1}\right)=4 \rho\left(x_{2}\right)$. From this the inequality (2) immediately follows.

Next we prove 2). The idea is similar, but simpler. It is known from page 109 of [6] that the bounded psh exhaustion function has an explicit form:

$$
\phi_{x_{0}}=\left(\tanh \frac{\sqrt{A} \rho_{0}}{2}\right)^{2}
$$

for any $x_{0} \in M$. Hence there exists a constant $b_{1}>0$ such that

$$
\widetilde{A}\left(x_{0}, b_{1}\right) \subset\left\{x \in M: \rho_{0}(x)<1\right\} .
$$

Repeating the argument as above, one can find a positive constant $C_{10}$ such that for any two points $x_{1}, x_{2} \in M$ with $\rho\left(x_{1}\right)=\rho\left(x_{2}\right)+3$, we have

$$
\operatorname{dist}_{\beta}\left(x_{1}, x_{2}\right) \geq C_{10} \text {, }
$$

from which the assertion immediately follows.

\section{REFERENCES}

[1] Z. Blocki and P. Pflug, Hyperconvexity and Bergman completeness, Nagoya J. Math. 151 (1998), 221-225. MR 2000b:32065

[2] B. Y. Chen, The Bergman metric on complete Kähler manifolds, preprint.

[3] B. Y. Chen and J. H. Zhang, Bergman exhaustivity, completeness and stability, Adv. Math. (Chinese) 29 (2000), 397-410. CMP 2001:08

[4] -, On Bergman completeness and Bergman stability, Math. Ann. 318 (2000), 517-526. CMP 2001:05

[5] K. Diederich and T. Ohsawa, An estimate for the Bergman distance on pseudoconvex domains, Ann. of Math. 141 (1995), 181-190. MR 95j:32039

[6] R. E. Greene and H. Wu, Function theory on manifolds which possess a pole, Lecture Notes in Mathematics 699, Springer-Verlag 1979. MR 81a:53002

[7] G. Herbort, The Bergman metric on hyperconvex domains, Math. Z. 232 (1999), 183-196. MR 2000i:32020

[8] L. Hörmander, An introduction to complex analysis in several variables, 3rd ed., North Holland 1990. MR 91a:32001

[9] J. Jost and K. Zuo, Vanishing theorems for $L^{2}$-cohomology on infinite covering of compact Kähler manifolds and applications in algebraic geometry, Comm. Geom. Anal. 8 (2000), 1-30. MR 2001f:32033

[10] M. Klimek, Extremal plruisubharmonic functions and invariant pseudodistances, Bull. Soc. Math. France. 113 (1985),123-142. MR 87d:32032

[11] S. Kobayashi, Geometry of bounded domains, Trans. Amer. Math. Soc. 92 (1959), 267-290. MR 22:3017

[12] -, On complete Bergman metrics, Proc. Amer. Math. Soc. 13 (1962), 511-513. MR 25:5192

[13] T. Ohsawa, Boundary behavior of the Bergman kernel function on pseudoconvex domains, Publ. RIMS, Kyoto Univ. 20 (1984), 897-902. MR 86d:32025

[14] R. Richberg, Stetige streng pseudoconvexe Funktionen, Math. Ann. 175 (1968), 257-286. MR 36:5386

[15] N. Sibony, A class of hyperbolic manifolds, In: Recent Developments in Several Complex Variables, Princeton Univ. Press (1981), 357-372. MR 83a:32022

[16] Y. T. Siu and S. T. Yau, Complete Kähler manifolds with nonpositive curvature of faster than quadratic decay, Ann. of Math. 105 (1977), 225-264; errata, ibid. 109 (1979), 621-623. MR 55:10719: MR 80h:32022 
[17] J. L. Stehlé, Fonctions plurisousharmoniques et convexité holomorphe de certains fibrés analytiques, Lecture Notes in Math. Séminaire P. Lelong, Springer-Verlag 474 (1973/1974), 155-179. MR 53:3368

[18] W. Zwonek, Completeness, Reinhardt domains and the method of complex geodesics in the theory of invariant functions, Dissertationes Mathematicae 388 (2000), 103 pp. MR 2001h:32016

Department of Applied Mathematics, Tongui University, Shanghai 200092, China

E-mail address: chenboy@online.sh.cn

Department of Mathematics, Fudan University, Shanghai 200433, China

E-mail address: zhangjhk@online.sh.cn 